\title{
Introduction to Information Transmission in a Neuron-Astrocyte
}

\author{
Jian Zhou ${ }^{1, a}$, Ning Cai ${ }^{1, b^{*}}$ and Lingjin Kong ${ }^{2, c}$ \\ ${ }^{1}$ College of Electrical Engineering, Northwest University for Nationalities, China \\ ${ }^{2}$ College of Journalism and Communication, Northwest University for Nationalities, China \\ azhoujianfrank@126.com, bcaining91@tsinghua.org.cn, ${ }^{\mathrm{c}}$ konglingjin928@126.com
}

\begin{abstract}
Keywords: Neuron-Astrocyte; Electrical activity; Information transmission; Time delay; Pattern formation
\end{abstract}

\begin{abstract}
In this review, the dynamics for neuron, neuronal network is introduced, for example, the mode transition in electrical activity, functional role of autapse connection, bifurcation verification in biological experiments, interaction between neuron and astrocyte, noise effect, coherence resonance, pattern formation and selection in network of neurons.
\end{abstract}

\section{Introduction}

Target wave can occur in a reaction-diffusion system and a two-dimensional array under local Heterogeneity or external periodical forcing in local area, and the continuous emitting wave can regulate the collective behavior of the media or coupled oscillators. Indeed, spiral wave also can be generated from heterogeneity after collision between ordered waves, for example, Ref. [1] reported the formation of spiral wave from fractal heterogeneity, Ref. [2] suggested that the emitting wave from heterogeneity induced by rotating electric field can be used to remove spatiotemporal turbulence in the media. Ref. [3] reported that spiral wave can also be induced in subexcitable media by applying external electric field.

The development of nonlinear science paves a reliable way to understand the transition of neuronal activities in neurons by using some reliable neuron model [4]. The dynamical properties of electric activities, stochastic resonance, coherence and synchronization of neurons have been investigated extensively [5]. Furthermore, the collective behaviors of neuronal network have also been investigated by detecting the spatiotemporal pattern selection, transition of spatial distribution [6], such as pulse wave [7] in a ring network, target wave ${ }^{\text {, }}$, spiral wave [7,8] in a two-dimensional array with different topological structures.

We find that repulsive and attractive forces increase with the enhancement of the level of heterogeneity for both parametric heterogeneity and impermeable inclusions. Notably, the attractive force is much larger than the repulsive force for all parameter values, suggesting that unpinning of the spiral tip is more difficult than pinning. Finally, the comparison shows that, for small-sized heterogeneities, the level and the size of heterogeneity both influence the forces remarkably. However, for large-sized heterogeneities, the forces are independent of the size, but are influenced by the level of the heterogeneity. This work may shed some light on the control or suppression of spiral waves

\section{Preliminaries}

To approach realistic model, diversity in time delay (and gain) in autapses are considered. It is found that target waves can be induced and then some target waves can convert into spiral waves and regulate the collective behaviors of network like pacemaker. Under appropriate coupling intensity, spiral wave can coexist with target waves, or perfect spiral waves can grow up and regulate the network synchronously. The free end of the emitted wave is generated by the REF itself from the rotating hyper-polarization instead of touching the refractory tail of the spiral in EP [9]. 
Consequently, the time window to apply pulse for successful liberation is much wider in REF than that in EP, which makes the REP more efficient. There are different heterogeneities in the cardiac tissue, such as blood vessels, fatty tissue, boundaries between regions of different fiber alignment directions, and intercellular clefts. Thus, during cardiac arrhythmia, various spiral waves with different frequencies, phase, and rotation direction may be pinned in various obstacles with different size. That means it is impossible to find an optimal rotating frequency, phase, and rotation direction of REP. A possible treatment of actual application is just letting the EP rotate so that to increase the efficiency, like that presented obviously. We address one point that the amplitude and duration of the REP is kept as that in EP, which indicates no additional energy is required while its efficiency is increased. We hope this strategy may improve manipulations with pinned spiral waves in related experiments, in cultured cardiac myocytes.

Notably, the attractive force is much larger than the repulsive force for all parameter values, suggesting that unpinning of the spiral tip is more difficult than pinning. The sampled time series for membrane potentials and correlation functions from three nodes are detected to analyze transition in electrical activities, so that collapse in network could be predicated before final collapse in regularity of network. It finds that the regular distribution in network could be destroyed completely with increasing the collapse area beyond certain threshold. The collapse in different parameters causes different deformation degrees for the network, and collapse in some critical parameters can induce rapid breakup of target wave in the network. The network distribution can also keep its regularity and robustness to collapse in certain parameter. In fact, the collapse of network cannot be predicated and prevented when the collapse in parameter is diffused with high speed (smaller diffusion period).For ecological system such as forest, fire or earthquake, even infectious disease in one region can ruin the balance of the ecological system, therefore, it is important to monitor some nodes in this area so that occurrence of disaster could be predicated as soon as possible.

\section{Information Transmission in a Neuron-Astrocyte Coupled Model}

Bursting-like Spikes. The effect of astrocyte serves an important function in the production of the BLSs. As previously mentioned, the excitatory coupling strength determines the information transmission from N1 to N2 significantly. Thus, we will identify the region of parameter 1 and gse, in which the BLSs are produced. Additionally, the IP3 production rate $\mathrm{rP}$ has been proven to be associated with the expression level of mGluRs in astrocytes. The enhanced production of IP3 corresponds to over-expressed mGluRs. Over-expression of mGluRs has been reported to facilitate the seizure-like oscillations in the neurons [10]. In upon study, three typical values of $\mathrm{rP}, 0.4,0.5$ and $0.8 \mathrm{mM} / \mathrm{s}$, are selected to represent the normal, intermediate, and enhanced expression level of mGluRs, respectively. The shadow regions are the parameter regions in which the BLSs can be found. First, the BLSs appear for an intermediate value of gse. Extremely large or small gse both make the calcium concentration approach a steady value less than $196.69 \mathrm{nM}$. In our model, the astrocyte fails to feedback to the neurons by Iastro when C is less than $196.69 \mathrm{nM}$. Thus, the BLSs are not produced. Second, only if 1 is larger than a critical value do BLSs appear. Thus, we can conclude that the existence of astrocyte is an important condition.

Time Delay and Information Distortion. Synaptic transmission is widely accepted to involve time delay attributed to the signal propagation time [11]. Theoretically, neuronal models with time delay have received considerable attention. Delay-induced coherent oscillation [12] is found in neuronal network as well as in other coupled systems. Delay enhanced synchronization may be relevant for neuronal networks to establish a concept of collective information processing in the presence of delayed information transmission. Our recent works find that delay cooperating with diversity can induce fruitful synchronization transitions. The model is as 
follows:

$$
\begin{aligned}
& C_{m} \frac{\partial V_{x}}{\partial t}=-g k n_{x}^{4}\left(V_{x}-v_{K}\right)-g N_{a} m_{x}^{3} h_{x}\left(V_{x}-v_{N a}\right)-g_{L}\left(V_{x}-V_{L}\right)+I_{a s k}+I_{e x}+I_{s x} \\
& \frac{\partial m_{x}}{\partial t}=\alpha_{m x}\left(1-m_{x}\right)-\beta_{m x} m_{x} \\
& \frac{\partial n_{x}}{\partial t}=\alpha_{n x}\left(1-n_{x}\right)-\beta_{n x} n_{x} \\
& \frac{\partial h_{x}}{\partial t}=\alpha_{h x}\left(1-h_{x}\right)-\beta_{h x} h_{x}
\end{aligned}
$$

Where $V_{x}$ denotes the transmembrane potential of $x$ th neuron $(x=1,2)$, and $n_{x}^{4}$ represents the fraction of open $\mathrm{Na}^{+}$channels and $m_{x}^{3} h_{x}$ represents the fraction of open potassium channels. The values of parameters are listed .The closing and opening rates of the gates are given by

$$
\begin{aligned}
& \alpha_{m x}=0.1 \frac{25-V_{x}}{e^{\frac{25-V_{x}}{10}}-1}, \beta_{m x}=4 e^{\frac{-V_{x}}{18}}, \\
& \alpha_{h x}=0.07 e^{\frac{-V_{x}}{20}}, \beta_{h x}=\frac{1}{e^{\frac{30-V_{x}}{10}}+1}, \\
& \alpha_{n x}=0.01 \frac{10-V_{x}}{e^{\frac{10-V_{x}}{10}}+1}, \beta_{n x}=0.125 e^{\frac{-V_{x}}{80}}
\end{aligned}
$$

Models. Distinguished pattern could be observed in chemical, physical and biological systems apart from thermodynamic equilibrium. For example, spiral wave can be developed in the excitable and oscillator media via Hopf bifurcation, and Turing patterns can be induced in the media via saddle node bifurcation. Spiral wave in the cardiac tissue is thought to be harmful signal which could be associated with arrhythmia, and breakup of spiral can cause rapid death of heart associated with ventricular fibrillation. Therefore, some schemes such as local pacing with periodical forcing, depolarization under electric field, and electric shock, even amplitude restriction are used to suppress the spiral waves and spatiotemporal chaos in the media. Spiral wave is also observed in the ecological system and cortex in the brain, some researchers designed different types of network in topology to reproduce the formation of spiral wave in the network, and potential mechanism for spirals formation and stability is extensively investigated. In general, target wave could be induced by imposing local periodical forcing, forcing with diversity, parameter in diversity for heterogeneity, self-feedback in close loop. Spiral wave can be developed by breaking the target wave or plane wave in the media. Sometimes, artificial defects are designed to emit stable pulse and target waves. It illustrates the distribution for artificial defects.

$$
\left\{\begin{array}{l}
\frac{d x}{d t}=y-a x^{3}+b x^{2}-z+I_{e x t}-k_{1} \rho(\varphi) x, \\
\frac{d y}{d t}=c-d x^{2}-y, \\
\frac{d z}{d t}=r[s(x+1.6)-z], \\
\frac{d \varphi}{d t}=x-k_{2} \varphi \\
\frac{d q(\varphi)}{d \varphi}=\rho(\varphi)
\end{array}\right.
$$




$$
i^{\prime}=\frac{d q(\varphi)}{d t}=\frac{d q(\varphi)}{d \varphi} \frac{d \varphi}{d t}=\rho(\varphi) V=k_{1} \rho(\varphi) x
$$

In addition, many measures can be applied to compare the eigenvalue properties for different brain states. Here, we used the largest eigenvalue, which is a useful and important index for predicting sudden changes in complex systems, to measure the change in dynamic brain network caused by visual stimulation. We calculated the relative change of the largest eigenvalue.

$$
R=\frac{\lambda_{\max }^{\text {visual }}-\lambda_{\max }^{\text {resting }}}{\lambda_{\max }^{\text {resting }}}
$$

\section{Discussion}

The diffusion period is marked as $1 \mathrm{t}$, which describes the transient period when the adjacent neurons outside of the collapsed area is affected and included into the collapsed area (with more nodes or size). For smaller $1 \mathrm{t}$, the collapsed area is increased with high speed. The number of nodes included into the collapsed area is marked as $\mathrm{S}$, and $\mathrm{Q}$ represents the state for neurons, for $\mathrm{Q}=0$, it means that the monitored node is in normal state without collapse in parameter while $Q=1$ indicates that the monitored node is in collapse state. The Euler forward algorithm is used for numerical calculation with time step $h=0.01$, the maximal number of nodes suffered from parameter collapse is about 60000. In a summary, diversity in time delay in autapses connected to neurons accounts for the emergence of target wave, and transition from target waves to spiral waves under appropriate gain and coupling intensity is associated to the collision between wave fronts of target waves. Under moderate coupling intensity, spiral wave can coexist with target wave or dominate the network completely, thus the collective behaviors of neurons can be in a regular way driven by the spiral wave or target wave like a pacemaker.

As mentioned above, the nervous system in brain contains billions of neurons, and different functional areas are formed. It is important to study the synchronization behaviors in neuronal network under different topologies or connection types. For brief review, readers can find some results in ref. [13] and some interesting results could also be found in the references therein. Time delay, channel blocking, and pacemaker driving are considered when the synchronization problem and stability in neurons are explored [14]. Furthermore, synchronization control for network becomes challengeable due to its potential application to preventing neuronal disease and multi-agent dynamics, Zhou et al. $[14,15]$ presented an analysis procedure for the controlled synchronization distributed coordinated control of the networked robotic systems formulated by Lagrange dynamics. For a practical way, pinning and impulsive control could effectively tame and stabilize the collective behaviors of network [15]. Indeed, much attention has been paid to the problems about network composed of complex variables, memristive neural network, and time-delayed network. Some excellent work of Cao and his collaborators could be found in refs and some references therein. Aiming at the key issues and bottleneck problems in the theory of coupled neural networks, a new system model, namely the nonlinear neuron-coupled network, was established, and an effective parameters estimation method was proposed based on the synchronization theory of the neural networks. Recurrent neural network is a special class of coupled network model stemming from the study of information transmission between neurons. It is a complex nonlinear parallel processing network composed of neurons, that information can be successfully transmitted and calculation becomes available due to the interaction between neurons. Recurrent neural networks have been extensively applied to different fields such as computational optimization, electronic engineering and control theory. 


\section{References}

[1] Jun Ma: EPL Europhysics Letters, Liberation of a Pinned Spiral Wave by a Rotating Electric Pulse.

[2] Jun Ma: Nonlinear Dynamics, (2014), Simulating Electric Activities of Neurons by Using Pspice.

[3] S.B. Liu, Y. Wu and J.J. Li: Nonlinear Dyn, (2013); 73: 1055-63, The Dynamic Behavior of Spiral Waves in Stochastic Hodgkin-Huxley Neuronal Networks with Ion Channel Blocks.

[4] Y. Wu, J.J. Li and S.B. Liu: Cogn Neurodyn, (2013); 7: 431-40, Noise-induced Spatiotemporal Patterns in Hodgkin-Huxley Neuronal Network.

[5] W. Wang, Perez G and Cerderia HA: Phys Rev E, (1993); 47(4):2893-8, Dynamical Behavior of the Firings in a Coupled Neuronal System.

[6] Jun Ma, Chunni Wang and Ying Wu: Plos One, (2014), Autapse-Induced Spiral Wave in Network of Neurons under Noise.

[7] L. Wang, Y.J. Zeng: Neurological Sci, (2013); 34.19771194?, Control of Bursting Behavior in Neurons by Autaptic Modulation.

[8] H.X. Qin, Jun Ma and C.N. Wang: Sci China Phys Mech Astro, (2014); 57: 1918-26, Autapse-induced Target Wave, Spiral Wave in Regular Network of Neurons.

[9] H.X. Qin, Jun Ma and W.Y. Jin: Sci China Tech Sci, (2014); 57: 936-46, Dynamics of Electric Activities in Neuron and Neurons of Network Induced by Autapse.

[10]H.T. Wang, Jun Ma and Y.L. Chen: Commun Nonlinear Sci Numer Simulat, (2014);19: 3242-54, Effect of an Autapse on the Firing Pattern Transition in a Bursting Neuron.

[11] Jun Ma, Y Wu and H.P. Ying: Chin Sci Bull, (2011); 56: 151-7, Channel Noise-induced Phase Transition of Spiral Wave in Networks of Hodgkin-Huxley Neurons.

[12] Wuyin Jin, Jun Ma and Z.B. Xie: Acta Phys Sin, (2013); 62:240507, Effect of Inhomogeneous Distribution of Ion Channels on Collective Electric Activities of Neurons in a Ring Network.

[13] J.Q. Zhang, C.D. Wang and M.S. Wang: Neurocomputing, (2011); 74: 2961-6, Firing Patterns Transition Induced by System Size in Coupled Hindmarsh-Rose Neural System.

[14]B. Jia, H.G. Gu: Acta Phys Sin, (2012); 61(24):240505 (in Chinese), Experimental Research on Synchronous Rhythms of Biological Network Composed of Heterogeneous Cells.

[15] Jun Ma, Y. Wu and N.J. Wu: Sci China Phys Mech Astro, Detection of Ordered Wave in the Networks of Neurons with Changeable Connection. 